\title{
An Iterative Method for Approximating Average Cost Optimal $(s, S)$ Inventory Policies $\left.{ }^{1}\right)$
}

\author{
By H. C. Tijms, Amsterdam ${ }^{2}$ )
}

Eingegangen am 21. Mai 1973

\begin{abstract}
Summary: This paper considers the dynamic inventory model with a discrete demand. There is a constant lead time, backlogging of excess demand, a fixed set-up cost, and holding and shortage costs whose negatives are unimodal. The criterion is the long-run average cost. A value iteration method with discount factor approaching to 1 is studied. This value iteration method supplies policies of the $(s, S)$ type and convergent upper and lower bounds on the minimal average cost. Further, the average cost of the $\left(s_{n}, S_{n}\right)$ policy found at the $n$-th iteration lies between the corresponding upper and lower bound. Also, for all $n$ sufficiently large the $\left(s_{n}, S_{n}\right)$ policy is average cost optimal. Computational considerations are given for the special case of linear holding and shortage costs.
\end{abstract}

\section{The Inventory Model and Preliminaries}

We consider an inventory model in which the demands for a single item in periods $1,2, \ldots$ are independent identically distributed discrete random variables. Let $\phi(j)$ be the probability of demand $j$ in a period $(j=0,1, \ldots)$. It is assumed that the demand distribution $\{\phi(j)\}$ has a finite, positive mean $\mu$. Any unfilled demand in a period is completely backlogged. At the beginning of each period the stock on hand and on order is reviewed. At each review an order may be placed for any positive integral amount of stock. An order placed at the beginning of period $t$ is delivered at the beginning of period $t+\lambda$, where $\lambda$ is a fixed non-negative integer. The demand in each period takes place after review and delivery (if any). The stock on hand and on order may take on any integral value, where a negative value indicates the existence of a backlog.

The following costs are considered. The cost of ordering $j$ units is $K \delta(j)$, where $K \geq 0, \delta(0)=0$, and $\delta(j)=1$ for $\left.j \geq 1^{3}\right)$. Let $g(j)$ be the (expected) holding and shortage costs in a period when $j$ is the stock on hand at the beginning of that period just after any additions to stock. It is assumed that $g(j)$ is non-negative. Future costs are not discounted. For any integer $k$, let

$$
L(k)=\sum_{j=0}^{\infty} g(k-j) \phi^{\lambda}(j),
$$

1) This paper is registered as Mathematical Centre Report BW 23/73.

$\left.{ }^{2}\right)$ Dr. Henk C. Tijms, Department of Operations Research, Mathematisch Centrum, Amsterdam.

${ }^{3}$ ) A linear purchase cost $c$ contributes an amount $c \mu$ to the average cost of any policy to be considered, so for the average cost criterion we may take $c=0$. 
where $\phi^{0}(0)=1, \phi^{0}(j)=0$ for $j \geq 1$ and, for $n \geq 1, \phi^{n}(j)$ is the $n$-fold convolution of $\phi(j)$ with itself, i. e., $\phi^{n}(j)$ is the probability of a cumulative demand $j$ in $n$ periods. We assume that $L(k)$ is finite for all $k$. Clearly, $L(k)$ represents the expected holding and shortage costs in period $t+\lambda$ when $k$ is the stock on hand and on order at the beginning of period $t$ just after ordering. The following conditions are imposed on $L(k)$ : (a) the function $-L(k)$ is unimodal, i. e., there is an integer $S_{0}$ such that $L(k) \leq L(k-1)$ for $k \leq S_{0}$ and $L(k+1) \geq L(k)$ for $k \geq S_{0}$; (b) $L(k)>K+L\left(S_{0}\right)$ for all $|k|$ sufficiently large. Define $r$ as the smallest integer such that

$$
L(r) \leq K+L\left(S_{0}\right),
$$

and let $R$ be the largest integer for which

$$
L(R) \leq K+L\left(S_{0}\right) .
$$

For the infinite period model an $(s, S)$ policy is a stationary policy of the following form: If, at review, the stock on hand and on order $i<s$, order $S-i$ units; otherwise do not order. Denote by $a(s, S)$ the long-run average expected cost per period when an $(s, S)$ policy is used. It is known that the quantity $a(a, S)$ is independent of the initial stock and is given by [Iglehart, 1963; Tijms, 1972; Veinott-Wagner, 1965]

$$
a(s, S)=\left\{L(S)+\sum_{k=0}^{s-s} L(S-k) m(k)+K\right\} /\{1+M(S-s)\},
$$
where $m(k)$ is defined by $m(k)=\phi(k)+\sum_{j=0}^{k} m(k-j) \phi(j)$ and $M(k)=\sum_{j=0}^{k} m(j)$,
$k \geq 0$. Let

$$
g=\min \{a(s, S) \mid s, S \text { integers, } s \leq S\} .
$$

A policy for controlling the stock is called average cost optimal when it minimizes the long run average expected cost per period for each initial stock. Under the conditions imposed on $L(k)$ we have that among the class of all possible policies there is an average cost optimal policy which is of the $(s, S)$ type [Johnson, 1968; Tijms, 1972] (this result was first proved by [Iglehart, 1963] for the case where $L(k)$ is convex, see also [Veinott-Wagner, 1965, pp. 530-531]). Hence the minimal average expected cost is independent of the initial stock and equals $g$. Moreover, there is an average cost optimal $(s, S)$ policy with $r \leq s \leq S \leq R$ [Johnson, 1968; Tijms, 1972; Veinott-Wagner, 1965]. Both in [Johnson, 1968] and in [VeinottWagner, 1965] an computational method for finding an average cost optimal $(S, S)$ policy has been given. These methods which are nearly identical to each other bear on the minimization of the quantity $a(s, S)$.

The purpose of this paper is to present a different approach which is based on the method of successive approximations. We shall treat a modified form of the value iteration method which is insensitive to any periodicity in the behaviour of the stock level. 
Let $\left\{\alpha_{n}, n=1,2, \ldots\right\}$ be a sequence with $0 \leq \alpha_{n} \leq 1$ for all $n$. Define $f_{0}(i)=0$ for any integer $i$, and, for $n=1,2, \ldots$, define for any integer $i$,

$$
f_{n}(i)=\inf _{k \geq i}\left\{K \delta(k-i)+L(k)+\alpha_{n} \sum_{j=0}^{\infty} f_{n-1}(k-j) \phi(j)\right\} .
$$

Then [Veinott, 1966], for $n=1,2, \ldots$,

$$
f_{n}(i)= \begin{cases}K+L\left(S_{n}\right)+\alpha_{n} \sum_{j=0}^{\infty} f_{n-1}\left(S_{n}-j\right) \phi(j) & \text { for } i<s_{n}, \\ L(i)+\alpha_{n} \sum_{j=0}^{\infty} f_{n-1}(i-j) \phi(j) & \text { for } i \geq s_{n},\end{cases}
$$

where $S_{n}$ is the smallest integer for which $G_{n}(k)=L(k)+\alpha_{n} \sum_{j=0}^{\infty} f_{n-1}(k-j) \phi(j)$ is minimal and $s_{n}$ is the smallest integer such that $G_{n}\left(s_{n}\right) \leq K+G_{n}\left(S_{n}\right)$. The result in (2) was first proved by Scarf [1960] for the case where $L(k)$ is convex (observe that for our model there is no difference between Veinott's formulation of the salvage cost in the finite period model and Scarf's one, since the linear purchase cost is zero). Further, we have [Veinott, 1966],

$$
r \leq s_{n} \leq S_{n} \leq R \text { for } n=1,2, \ldots
$$

\section{Approximations for Average Cost Optimal $(s, S)$ Policies}

In this section we shall prove that the recursion in (2) supplies lower and upper bounds $L_{n}$ and $U_{n}$ such that $L_{n} \leq g \leq a\left(s_{n}, S_{n}\right) \leq U_{n}$ for all $n$. Moreover, under certain conditions, both $L_{n}$ and $U_{n}$ converge as $n \rightarrow \infty$ to the minimal average cost $g$, and for all $n$ sufficiently large the $\left(s_{n}, S_{n}\right)$ policy is average cost optimal.

We introduce the following conditions.

\section{Condition 1:}

(i) $0<\alpha_{n}<1$ for $n=1,2, \ldots$; (ii) $\alpha_{n} \rightarrow 1$ as $n \rightarrow \infty$;

(iii) $\alpha_{1} \alpha_{2} \ldots \alpha_{n} \rightarrow 0$ as $n \rightarrow \infty$; and (iv) $\sum_{j=2}^{n}\left(\alpha_{n} \alpha_{n-1} \ldots \alpha_{j}\right)\left|\alpha_{j}-\alpha_{j-1}\right| \rightarrow 0$ as $n \rightarrow \infty$.

Condition 2:

(i) $\alpha_{n}=1$ for $n=1,2, \ldots$; and (ii) $\phi(i)>0$ for some $i>R-r$.

Remark 1:

It is readily verified that Condition 1 is satisfied when $\alpha_{n}=1-(n+1)^{-b}$ for all $n$ provided that $\frac{1}{2}<b \leq 1$ (see also [Hordijk-Tijms, 1973a]).

Given the sequence $\left\{\alpha_{n}\right\}$, we define

$$
\gamma_{0}=0 \quad \text { and } \gamma_{n}=1+\alpha_{n} \gamma_{n-1} \text { for } n=1,2, \ldots \text {. }
$$

Observe that $\gamma_{n}=n$ for all $n$ when $\alpha_{n}=1$ for all $n$. We shall need the following theorem. 
Theorem 1:

If Condition 1 or Condition 2 is satisfied, then

$$
\lim _{n \rightarrow \infty}\left\{f_{n}(i)-\gamma_{n} g\right\} \quad \text { exists and is finite for all } i=r-1, \ldots, R .
$$

Proof:

Let us define a Markovian decision problem which is closely related to the inventory model under consideration. Consider a dynamic system which at times $t=1,2, \ldots$ is observed to be in one of $R-r+2$ states labeled by $i=r-1, \ldots, R$. After observing state $i$, an action $k$ is chosen from the set $A(i)$ of possible actions, where $A(i)=\{i, i+1, \ldots, R\}$. If at time $t$ the system is in state $i$ and action $k$ is chosen, then an expected cost $c_{i}^{k}=K \delta(k-i)+L(k)$ is incurred and at time $t+1$ the system will be in state $j$ with probability $p_{i j}^{k}$, where $p_{i j}^{k}=\phi(k-j)$ for $j \neq r-1$ and $p_{i, r-1}^{k}=\sum_{h>k-r} \phi(h)$ with $\phi(m)=0$ for $m<0$.

By (2) and (3) we have $f_{n}(i)=f_{n}(r-1)$ for all $i<r$ and $n \geq 0$, so, by (1) and (2),

$$
f_{n}(i)=\min _{k \in \boldsymbol{A}(i)}\left\{c_{i}^{k}+\alpha_{n} \sum_{j=r-1}^{R} f_{n-1}(j) p_{i j}^{k}\right\} \quad \text { for } \quad r-1 \leq i \leq R \quad \text { and } n \geq 1 \text {. }
$$

Further, using the fact that for the inventory model there is an average cost optimal policy of the $(s, S)$ type with $r \leq s \leq S \leq R$, it is easily seen that for the above Markovian decision problem the minimal average expected cost per unit time is independent of the initial state and equals $g$. It now follows from Theorem 1 in [Hordijk-Tijms, 1973a] that under Condition 1 the result (5) holds. Assume now that Condition 2 is satisfied. Then, for any stationary policy $f$ adding to each state $i$ an action $f(i) \in A(i)$, the associated Markov chain $\left(\left(p_{i j}^{f(i)}\right)\right)$ has a single recurrent class and has no periodic states. It now follows from (Lanery [1967], Theorem 3 on p. 43) that $f_{n}(i)-n g$ has a finite limit as $n \rightarrow \infty$ for all $r-1 \leq i \leq R$ (see also [Odoni, 1969]). This ends the proof.

The proof of the next theorem is an adaptation of proofs given in [Hordijk-Tijms, 1973a, 1973b]; for the case where $\alpha_{n}=1$ for all $n$ see also [Hastings, 1971] and [Odoni, 1969].

\section{Theorem 2:}

For any $n \geq 1$, let

$$
\begin{gathered}
L_{n}=\min \left\{f_{n}(i)-\alpha_{n} f_{n-1}(i) \mid r_{n}-1 \leq i \leq R\right\}, \\
U_{n}=\max \left\{f_{n}(i)-\alpha_{n} f_{n-1}(i) \mid r_{n}-1 \leq i \leq S_{n}\right\},
\end{gathered}
$$

where $r_{1}=s_{1}$ and $r_{n}=\min \left(s_{n-1}, s_{n}\right)$. Then

(a) $L_{n} \leq g \leq a\left(s_{n}, S_{n}\right) \leq U_{n}$ for all $n \geq 1$.

If Condition 1 or Condition 2 is satisfied, then

(b) Both $L_{n}$ and $U_{n}$ converge as $n \rightarrow \infty$ to $g$.

(c) For all $n$ sufficiently large the $\left(s_{n}, S_{n}\right)$ policy is average cost optimal. 
Proof:

We first introduce some notation. Let $F=\{(s, S) \mid r \leq s \leq S \leq R\}$. For any $(s, S)$ policy from $F$, define for $i, j=r-1, \ldots, S$

$$
\begin{gathered}
p_{i j}(s, S)=\phi(i-j),(i \geq s, j \neq r-1), p_{i, r-1}(s, S)=\sum_{k>i-r} \phi(k),(i \geq s), \\
p_{i j}(s, S)=\phi(S-j),(i<s, j \neq r-1), \quad \text { and } \quad p_{i, r-1}(s, S)=\sum_{k>S-r} \phi(k),(i<s) .
\end{gathered}
$$

Denote by $\left\{\pi_{i}(s, S), i=r-1, \ldots, S\right\}$ the unique stationary probability distribution of the Markov matrix $\left(\left(p_{i j}(s, S)\right)\right)$. Let $c_{i}(s, S)=K+L(S)$ for $i<s$, and let $c_{i}(s, S)=L(i)$ for $i \geq s$. Clearly, for all $j=r-1, \ldots, S$,

$$
\pi_{j}(s, S)=\sum_{i=r-1}^{S} \pi_{i}(s, S) p_{i j}(s, S), \quad \text { and } a(s, S)=\sum_{i=r-1}^{S} c_{i}(s, S) \pi_{i}(s, S) .
$$

For part (a), fix $n \geq 1$. By (1) and (2) we have for any $(s, S) \in F$,

$$
f_{n}(i) \leq \begin{cases}K+L(S)+\alpha_{n} \sum_{j=0}^{\infty} f_{n-1}(S-j) \phi(j) & \text { for } \quad r-1 \leq i<s, \\ L(i)+\alpha_{n} \sum_{j=0}^{\infty} f_{n-1}(i-j) \phi(j) & \text { for } \quad s \leq i \leq S,\end{cases}
$$

with equality for all $i$ when $s=S_{n}$ and $S=S_{n}$. Since $f_{n-1}(i)=f_{n-1}(r-1)$ for $i<r$, we can write (7) in the equivalent form

$$
f_{n}(i) \leq c_{i}(S, S)+\alpha_{n} \sum_{j=r-1}^{S} f_{n-1}(j) p_{i j}(s, S) \quad \text { for } \quad r-1 \leq i \leq S,
$$

with equality for all $i$ when $s=s_{n}$ and $S=S_{n}$. By (2) we have $f_{n}(i)-\alpha_{n} f_{n-1}(i)$ is constant for $i<r_{n}$, so $f_{n}(i) \geq \alpha_{n} f_{n-1}(i)+L_{n}$ for all $i \leq R$. Choose now an $(s, S)$ policy from $F$. Then, by (8),

$\alpha_{n} f_{n-1}(i)+L_{n} \leq c_{i}(s, S)+\alpha_{n} \sum_{j=r-1}^{S} f_{n-1}(j) p_{i j}(s, S)$ for $\quad r-1 \leq i \leq S$.

Now multiply both sides of (9) by $\pi_{i}(s, S)$ and sum over $i$. Using (6), we find

$$
\alpha_{n} \sum_{i} f_{n-1}(i) \pi_{i}(s, S)+L_{n} \leq a(s, S)+\alpha_{n} \sum_{j} f_{n-1}(j) \pi_{j}(s, S),
$$

so $L_{n} \leq a(s, S)$. This implies $L_{n} \leq g$, since $g=a(s, S)$ for some $(s, S) \in F$. Consider now the $\left(s_{n}, S_{n}\right)$ policy. When $s=s_{n}$ and $S=S_{n}$ the equality sign holds in (8) for all $i$. Further, $f_{n}(i) \leq \alpha_{n} f_{n-1}(i)+U_{n}$ for all $i \leq S_{n}$. Hence

$$
\alpha_{n} f_{n-1}(i)+U_{n} \geq c_{i}\left(s_{n}, S_{n}\right)+\alpha_{n} \sum_{j=r-1}^{S_{n}} f_{n-1}(j) p_{i j}\left(S_{n}, S_{n}\right) \text { for } \quad r-1 \leq i \leq S_{n},
$$

from which we derive in the same way as above that $U_{n} \geq a\left(s_{n}, S_{n}\right)$.

(b) This assertion is an immediate consequence of (4) and (5).

(c) The proof of this assertion is a minor modification of proofs given in (HordijkTijms [1973a], Theorem 2(b)) and (Odoni [1969], Theorem $1(\mathrm{v})$ ) and is omitted. 
Remark 2:

The assumption of a linear purchase cost zero enabled it to reduce the inventory model with a denumerable number of possible stock levels to a finite state Markovian decision model. However, for the average cost criterion it is no restriction to assume that the linear purchase cost equals zero (see the footnote in section 1). Of course, the minimal total expected cost in the finite period inventory model depends on the value of the linear purchase cost. The case of a linear purchase cost $c>0$ has been studied in [Hordijk-Tijms, 1973b] under the assumptions that $L(k)$ is convex, $\alpha_{n}=1$ for all $n$ and $\phi(i)>0$ for all $i$ sufficiently large. It has been shown in [Hordijk-Tijms, 1973b] that under these conditions Theorem 2 holds also provided that $K \delta(k-i)$ is replaced by $c .(k-i)+K \delta(k-i)$ in (2), $L(k)$ is replaced by $c k+L(k)$ in the relations defining $s_{n}$ and $S_{n}$, and the term $c \mu$ is added to the right side of the expression for $a(S, S)$ given in section 1.

\section{Computational Considerations}

In this section we give some general findings for the convergence of the algorithm (2) for the cases $\alpha_{n}=1$ and $\alpha_{n}=1-(n+1)^{-b}$ where $\frac{1}{2}<b \leq 1$. We assume that the holding and shortage costs in a period are given by $h \cdot \max (i, 0)-p \cdot \min (i, 0)$ when $i$ is the stock on hand at the end of that period, where $h>0$ and $p>0$. Then [cf. Veinott-Wagner, 1965],

$$
L(k)=p\{(\lambda+1) \mu-k\}+(h+p) \sum_{j<k} \Phi^{\lambda+1}(j),
$$

where $\Phi^{\lambda+1}(j)=0$ for $j<0$ and $\Phi^{\lambda+1}(j)=\phi^{\lambda+1}(0)+\cdots+\phi^{\lambda+1}(j)$ for $j \geq 0$. It will be clear that the knowledge of the distribution function $\Phi^{\lambda+1}(\cdot)$ is sufficient to compute the bounds $r$ and $R$. The functions $L(k)$ and $f_{n}(k)$ need be computed only for $r-1 \leq k \leq R$ (see (2) and (3)). We have investigated two types of demand distribution $\phi(\cdot)$ : An arbitrary distribution with $\phi(j)>0$ for finitely many values of $j$, and a Poisson distribution.

In the computer program the algorithm was stopped when $\left(U_{n}-L_{n}\right) / L_{n} \leq \varepsilon$, which implies $a\left(s_{N}, S_{N}\right) \leq(1+\varepsilon) g$ where $N$ is defined as the first value of $n$ for which the convergence criterion $\left(U_{n}-L_{n}\right) / L_{n} \leq \varepsilon$. The number $\varepsilon$ was chosen equal to $0.05,0.01$, or 0.005 .

Considerable computer experimentation has demonstrated that for the algorithm with $\alpha_{n}=1-(n+1)^{-b}$ the convergence of $\left(U_{n}-L_{n}\right) / L_{n}$ becomes substantially worse as $b$ decreases, where $\frac{1}{2}<b \leq 1$. In most examples tested where the algorithm with $\alpha_{n}=1$ achieved convergence the number of iterations required for this algorithm was considerably less than the number of iterations required for the algorithm with $\alpha_{n}=1-(n+1)^{-1}$. For the algorithm with $\alpha_{n}=1$ the criterion $\left(U_{n}-L_{n}\right) / L_{n}$ exhibited a strong tendency to decrease exponentially with the number of iterations when the demand is Poisson distributed. This agrees with theoretical results in [White, 1963]. For the algorithm with 
$\alpha_{n}=1-(n+1)^{-b}$ our numerical results indicate that $\left(U_{n}-L_{n}\right) / L_{n}=0\left(1 / n^{2 b-1}\right)$, as might be expected from theoretical results in [Hordijk-Tijms, 1973a]. Finally, there did not seem to be any clear relation between the number of iterations required for convergence and the value of $R-r+1$; in fact the convergence depends on the probability $\sum_{k>R-r} \phi(k)$.

In table 1 we summarize some numerical results for two examples with an arbitrary demand distribution, where the other parameters are given by (cf. [Wagner, p. A 32]),

$$
K=24, h=4, p=10, \text { and } \lambda=0 .
$$

Except the results in the columns with entry $N^{\prime}$, the results in table 1 refer to the algorithm with $\alpha_{n}=1-(n+1)^{-1}$. The integer $N^{\prime}$ denotes the number of iterations required for the algorithm with $\alpha_{n}=1-(n+1)^{-0.6}$. For each case from table 1 the latter algorithm produced the same policy at the last iteration as the algorithm with $\alpha_{n}=1-(n+1)^{-1}$ did at the final iteration. For the example with $\phi(3)=1$ the algorithm with $\alpha_{n}=1$ leads to $L_{n}=12, U_{n}=24$ for $n \geq 4,\left(s_{n}, S_{n}\right)=(1,3)$ for $n$ odd and $\left(s_{n}, S_{n}\right)=(2,6)$ for $n$ even. We have $a(1,3)=24$ and $a(2,6)=18$; the policy $(2,6)$ is average cost optimal. For the example with $\phi(4)=\phi(5)=0.5$ the algorithm with $\alpha_{n}=1$ leads to $L_{n}=19.5, U_{n}=26$ for $n \geq 3,\left(s_{n}, S_{n}\right)=(2,5)$ for $n$ odd and $\left(s_{n}, S_{n}\right)=(3,9)$ for $n$ even. We have $a(2,5)=26$ and $a(3,9)=22.75$; the policy $(3,9)$ is average cost optimal

Table 1. $\alpha_{n}=1-(n+1)^{-1}$ and $\alpha_{n}=1-(n+1)^{-0.6}$

\begin{tabular}{c|c|c|c|c|c|c|c}
\hline \multicolumn{3}{c|}{$\phi(3)=1 ; r=1, R=11$} & \multicolumn{4}{c}{$\phi(4)=\phi(5)=0.5 ; r=2, R=11$} \\
\hline$\varepsilon$ & $N$ & $N^{\prime}$ & $\left(s_{N}, S_{N}\right)$ & $\varepsilon$ & $N$ & $N^{\prime}$ & $\left(s_{N}, S_{N}\right)$ \\
0.05 & 27 & 249 & $(2,6)$ & 0.05 & 20 & 169 & $(3,9)$ \\
0.01 & 133 & $>500$ & $(2.6)$ & 0.01 & 106 & $>500$ & $(3,9)$ \\
0.005 & 267 & & $(2,6)$ & 0.005 & 211 & & $(3,9)$ \\
\hline
\end{tabular}

In the tables 2 and 3 we summarize some numerical results for a number of examples with a demand distribution of the Poisson type, where the other parameters are given by (cf. [Wagner-O'Hagan-Lundh, 1965, p. 697]),

$$
K=64, h=1, p=9, \text { and } \lambda=0 .
$$

The cases I, II and III correspond to $\varepsilon=0.05, \varepsilon=0.01$ and $\varepsilon=0.005$. The results in the tables 2 and 3 refer to the algorithm with $\alpha_{n}=1$, except the results in the rows with entry $N^{\prime}$. The integer $N^{\prime}$ denotes the number of iterations required for the algorithm with $\alpha_{n}=1-(n+1)^{-1}$. When $\varepsilon=0.05$ the latter algorithm produced the policies $(2,23)$ and $(6,36)$ for $\mu=4$ and $\mu=9$ at the last iteration; for each other case we found the same policy as we did for the algorithm with $\alpha_{n}=1$. Each policy from the tables 2 and 3 is average cost optimal, except the policy $(12,51)$ for $\mu=16$. 
Table 2. $\alpha_{n}=1$ and $\alpha_{n}=1-(n+1)^{-1}$

\begin{tabular}{c|c|c|c|c|c|c}
\hline & $\mu$ & 1 & 2 & 4 & 9 & 16 \\
\hline \multirow{2}{*}{ Case I } & $r, R$ & $-6,67$ & $-5,68$ & $-3,71$ & 2,78 & 9,87 \\
& $N$ & 75 & 39 & 24 & 20 & 20 \\
& $N^{\prime}$ & 118 & 83 & 59 & 39 & 29 \\
& $\left(s_{N}, S_{N}\right)$ & $(0,11)$ & $(1,16)$ & $(2,24)$ & $(6,37)$ & $(12,51)$ \\
\hline \multirow{2}{*}{ Case II } & $N$ & 82 & 43 & 36 & 32 & 32 \\
& $N^{\prime}$ & $>500$ & 410 & 290 & 193 & 146 \\
& $\left(S_{N}, S_{N}\right)$ & $(0,11)$ & $(1,16)$ & $(2,24)$ & $(6,37)$ & $(12,52)$ \\
\hline \multirow{2}{*}{ Case III } & $N$ & 85 & 45 & 42 & 37 & 38 \\
& $N^{\prime}$ & $>500$ & $>500$ & $>500$ & 386 & 291 \\
& $\left(S_{N}, S_{N}\right)$ & $(0,11)$ & $(1,16)$ & $(2,24)$ & $(6,37)$ & $(12,52)$ \\
\hline
\end{tabular}

Table 3, $\alpha_{n}=1$ and $\alpha_{n}=1-(n+1)^{-1}$

\begin{tabular}{c|c|c|c|c|c|c}
\hline & $\mu$ & 20 & 25 & 36 & 49 & 64 \\
\hline \multirow{2}{*}{ Case I } & $r, R$ & 13,92 & 17,98 & 28,110 & 41,125 & 56,142 \\
& $N$ & 17 & 29 & 113 & 75 & 3 \\
& $N^{\prime}$ & 26 & 27 & 21 & 18 & 17 \\
& $\left(s_{N}, S_{N}\right)$ & $(15,62)$ & $(20,56)$ & $(30,79)$ & $(42,106)$ & $(56,74)$ \\
\hline \multirow{2}{*}{ Case II } & $N$ & 34 & 69 & 200 & 170 & 3 \\
& $N^{\prime}$ & 130 & 118 & 104 & 91 & 82 \\
& $\left(s_{N}, S_{N}\right)$ & $(15,62)$ & $(20,56)$ & $(30,79)$ & $(42,106)$ & $(56,74)$ \\
\hline \multirow{2}{*}{ Case III } & $N$ & 41 & 86 & 237 & 211 & 3 \\
& $N^{\prime}$ & 261 & 236 & 207 & 182 & 164 \\
& $\left(s_{N}, S_{N}\right)$ & $(15,62)$ & $(20,56)$ & $(30,79)$ & $(42,106)$ & $(56,74)$ \\
\hline
\end{tabular}

Finally, we note that it seems difficult to choose a stop criterion based on reiteration of policies. Several examples were encountered in which $s_{n}$ and $S_{n}$ did not change for a number of successive values of $n$ whereas no convergence in policy was achieved. In table 4 we give an example of this phenomenon for the

Table 4. $\mu=4, \alpha_{n}=1-(n+1)^{-1}$

\begin{tabular}{c|c|c}
\hline$n$ & $s_{n}, S_{n}$ & $a\left(s_{n}, S_{n}\right)$ \\
\hline $8-9$ & 2,20 & 22.483 \\
$10-11$ & 2,21 & 22.325 \\
$12-23$ & 2,22 & 22.224 \\
$24-168$ & 2,23 & 22.173 \\
$\geq 169$ & 2,24 & 22.166 \\
\hline
\end{tabular}


algorithm with $\alpha_{n}=1-(n+1)^{-1}$, where the demand is Poisson distributed with mean $\mu=4$ and the other parameters are given in (10).

Remark 3:

In this paper we have presented for the dynamic inventory model a modified form of the value iteration method which removes any asymptotic fluctuations. The computational considerations demonstrate that in the aperiodic case the value iteration method with $\alpha_{n}=1$ for all $n$ is superior to the value iteration method with discount factor approaching to 1 . In its turn, however, the former method will need in general more computing time than the algorithms given in [Johnson, 1968] and [Veinott-Wagner, 1965] (cf. [Riess, 1971]).

\section{Acknowledgement}

I am indebted to Mr. R. van der Horst, computer programmer at the Mathematisch Centrum, for writing the computer program.

\section{References}

Hastings, N. A. J.: Bounds on the Gain of Markov Decision Processes, Operations Research 19 , $240-243,1971$

Hordijk, A., and H. C. Tijms: A Modified Form of the Iterative Method of Undiscounted Dynamic Programming, Report BW 21/73, Mathematisch Centrum, Amsterdam 1973a (to appear in The Annals of Mathematical Statistics).

- : Convergence Results and Approximations for Optimal $(s, S)$ Policies, Report BW 22/73, Mathematisch Centrum, Amsterdam 1973b (to appear in Management Sciences).

Iglehart, D. L.: Dynamic Programming and Stationary Analysis of Inventory Problems, Chap. 1 in: H. Scarf, D. Gilford, and M. Shelly (eds.): Inventory Models and Techniques, Stanford Univ. Press, Stanford, Calif. 1963.

Johnson. E. L. : On (s.S) Policies. Management Science 9. 80-101. 1968.

Lanery, E.: Etude Asymptotique des Systèmes Markoviens à Commande, Revue Française d'Informatique et de Recherche Opèrationnelle 1, No. 5, 3-56, 1967.

Odoni, A. R.: On Finding the Maximal Gain for Markov Decision Processes, Operations Research 17. $857-860.1969$

Riess, G.: Vergleichende Untersuchung zur Bestimmung der Optimalen Parameter von ( $s, S)$-Politiken. Diplomarbeit TU München, Abteilung für Angewandte Mathematik 1971.

Scarf, H.: The Optimality of $(S, s)$ Policies in the Dynamic Inventory Problem, Chap. 13 in: $K . J$. Arrow, S. Karlin, and P. Suppes (eds.): Mathematical Methods in the Social Sciences, Stanford Univ. Press, Stanford, Calif. 1960.

Tijms, H. C. : Analysis of $(s, S)$ Inventory Models, Mathematical Centre Tract No. 40, Mathematisch Centrum. Amsterdam 1972.

Veinott, A. F. Jr.: On the Optimality of $(s, S)$ Inventory Policies: New Conditions and a New Proof Journal Siam Applied Mathematics 14, 1067-1083, 1966.

Veinott, A. F., Jr., and H. M. Wagner: Computing Optimal $(s, S)$ Policies, Management Science 11. $525-552.1965$

Wagner, H. M., with O'Hagan, M., and B. Lundh: An Empirical Study of Exactly and Approximately Optimal Inventory Policies, Management Science 11, 690-723, 1965.

Wagner, H. M. : Principles of Operations Research, Prentice-Hall, New York 1969.

White, D. J.: Dynamic Programming, Markov Chains, and the Method of Successive Approximations, Journal of Mathematical Analysis and Applications 6, 373-376, 1963. 